\title{
Challenges of Participatory Qualitative Research in a Malaysian and Australian Hospital
}

\author{
Kate Bishop ${ }^{1}$, Ismail Said ${ }^{2}$ \\ ${ }^{1}$ Faculty of Built Environment, University of New South Wales, Australia \\ ${ }^{2}$ Faculty of Built Environment, Universiti Teknologi MARA, Malaysia
}

k.bishop@unsw.edu.au

\begin{abstract}
Research in paediatric nursing, paediatric psychology, health care management and environmentbehaviour studies suggest that there are many obstacles in completing qualitative research in health care settings with children and young people. As a result, the evidence from this kind of research is limited. Both authors have recently completed participatory qualitative studies with children and young people in paediatric environments, both in Malaysia and Australia. These will be briefly described and some of the practical and methodological challenges that each researcher experienced will be discussed. Each challenge has strong implications and potential impact on the research quality; and also on the children and young people's experience of research.
\end{abstract}

Keywords: qualitative, children, health care, research

eISSN 2514-751X @ 2017 The Authors. Published for AMER ABRA by e-International Publishing House, Ltd., UK.. This is an open access article under the CC BY-NC-ND license (http://creativecommons.org/licenses/by-ncnd/4.0/). Peer-review under responsibility of AMER (Association of Malaysian Environment-Behaviour Researchers), ABRA (Association of Behavioural Researchers on Asians) and cE-Bs (Centre for EnvironmentBehaviour Studies), Faculty of Architecture, Planning \& Surveying, Universiti Teknologi MARA, Malaysia.

https://doi.org/10.21834/aje-bs.v2i4.205 


\subsection{Introduction}

Twenty years after the United Nations Convention on the Rights of the Child (1989) which enshrined children's right to participate in processes that have the capacity to affect their lives, researching children's and young people's experience of hospital and health care environments is still extremely difficult. There are some overarching challenges. A reasonable level of scepticism and misunderstanding still persists in the medical circles about qualitative research and this type of research still struggles to receive funding because its outcomes are not predictable. Beyond these constraints, the real challenges are linked to the sample population. For the researcher, the challenges include acquiring ethical approval, acquiring access, negotiating gatekeepers, recruiting participants and negotiating the medical culture of a hospital and its impact on the research process (Alderson, 1993, 1995; Bricher, 1999, 2000; Morison et al., 2000; Stalker et al., 2004).

Both the authors have recently completed participatory qualitative studies with children and young people in paediatric environments, in Malaysia (Said, 2008) and Australia (Bishop, 2008). The methodology of these studies varied and included both a phenomenological study and a case study. These will be briefly described, outlining the practical and methodological challenges that each author experienced. Each challenge has strong implications and potential impact on the research and its quality; to the children and young people's experience of research.

Some of these challenges include: maximising children's participation and maintaining data quality. Making the research processes simple and accessible for children and young people, while at the same time producing rigorous research results, is a challenge. A second challenge includes supporting the participation of children and young people in the research. Children's engagement in research requires a substantial time investment to ensure they comprehend the research process and are truly 'on the journey' with researchers. Participatory research is messy because it needs to be flexible and adaptable; is complex - logistically, methodologically and ethically, and resource intensive. These things are challenges for all participatory research but they are substantially increased by the context of a hospital where there are overlays of organisational culture, time, access constraints and competing agendas. Some of these challenges will be discussed as they are experienced in the two different cultural contexts.

\subsection{Literature Review}

Since the early 1990s, there has been increasing interest in research on children's and young people's experience of hospitalisation. This research has reflected the participatory philosophy for the most part with authors articulating the value of gaining children's and young people's insight into their experience (Carney et al., 2003; Miller et al., 1998; Sandbaek, 1999; Sartain et al., 2000; Smith \& Callery, 2005). There remains, however, very little participatory research on children's and young people's experience of hospitalisation. 
Participatory research began tentatively in the medicine and health science disciplines. In early research into children's experience in health care contexts, children's voices were often obscured by the voices of the surrounding adults (Eiser, 2000). However, more recent studies have used sample groups of children and young people as their only sample group (Miller et al., 1998; Morison et al., 2000; Hutton, 2002, 2005). This reflects a confidence in children's abilities and competence that has developed in response to research with children and young people (Mauthner, 1997). Participatory research with children and young people in this context does not provide a complete picture as yet but within this research there are persistent themes and key methodological recommendations. For this paper, only the key methodological considerations that have been identified for conducting participatory research in a health care setting will be stated as they pertain to some of the challenges relevant to this discussion. These include:

- $\quad$ The need to negotiate a hierarchy of 'gatekeepers' to access children in hospitals and the difficulty of this (Stalker et al., 2004)

- $\quad$ Managing ethical considerations, power relations between researcher and participants and empowering children in the research process (Alderson, 1993, 1995; Bricher, 1999, 2000; Morison et al., 2000)

- $\quad$ The importance of the social and physical context of the interviews and the need for privacy (Mauthner, 1997; Miller, 2000; Morison et al., 2000)

- $\quad$ The challenging nature of the research topic for participants and the perceived benefits and threats of the research (Morison et al., 2000)

- The influence of parents or 'significant adults' on children's participation (Morison et al., 2000; Sandbaek, 1999)

All of these considerations remain relevant to participatory research in health care contexts and most of these challenges are relevant to the experiences of both Said and Bishop in their respective research projects.

\subsection{Methodology}

The methodology of Bishop's (2008) study consisted of a participatory qualitative case study completed with children and young people aged 9-18 years, at the Children's Hospital at Westmead in Sydney, Australia. The aims of the research were firstly to understand what constitutes a supportive paediatric setting from children's and young people's perspectives; secondly to describe the roles of the physical environment in children's feeling of wellbeing; and thirdly, to illustrate the value of participatory research to health care design. 
The study was comprised of four stages. Fifty five children and young people aged 7-18 years were involved in the four stages of the study. Twenty four of these were involved in pilot studies, including nine boys aged 7-16 years and 15 girls aged 7-18 years. Thirty one participants were involved in the main study, including 13 boys aged $9-17$ years and 18 girls aged 10-18 years. Participants in the main study had to have been admitted to the hospital for at least seven days at the time they were interviewed. Within the main study, children and young people were asked to complete a single interview which consisted of three tasks. Task $A$ included an informal discussion centred on a set of photographs of the Hospital environment, taken by participants in the second pilot study, Part A. Task B involved answering a set of 30 questions spanning the possible domains and dimensions of children's experience of hospitalisation and well-being derived from both the literature and the findings from the pilot studies. Task $C$ involved a game task which functioned as a consistency check within each interview.

The interviews resulted in three sets of data including two sets of narrative data from tasks $A$ and $B$ which were analysed using concept mapping and thematic analysis techniques; and the game task results, which required tallying. The results from each of the three data sets were triangulated to create preliminary findings. These preliminary findings were developed into a series of statements and summaries that were then taken back to a group of six patients who were representative of the profile of participants in the main study, for further discussion as part of a member-checking task, before the final conclusions were reached.

Said's (2008) study used a phenomenological approach to study children's use and response to, a purpose-built hospital garden at Batu Pahat Hospital, in the state of Johor, Malaysia. In this study the focus was on understanding the interaction of 31 middle childhood patients, aged 6-12 years with the properties of a hospital garden as well as with peers playing in the garden. The garden was located beside the paediatric ward and was designed by the researcher and built with the funds from his research grant.

In a phenomenological study, the researcher aims to develop a first person understanding and perspective on the phenomenon being studied (Creswell, 1998). In a period of a week, the researcher played the role of a gardener to make the children aware that he was preparing a place for them to play. He was always clear in his mind that he was in the children's context and not his own and that he would not intervene or control the children's activities. Behaviour mapping was used to plot children's use of the garden and open-ended interviews were used to understand their responses to the garden. The former reflected the overt (externalised) responses of the children playing with the garden features and micro climate of a tropical garden. The latter revealed the covert (internalised) responses, reflecting the children's perceptions of the garden.

The interview was conducted in a conversational and relaxed style. For example, an interview was completed with two nephritic patients when both of them were curious to look at the camera, tape recorder, maps and questionnaires left by the researcher in a pavilion of the garden. To maintain their attention, the tape recorder was given to them to hold and 
then it was rewound for them to listen to their recorded conversation. The interview lasted only seven minutes initially and was resumed when the children came back from the ward or from other areas of the garden. Interview sessions were completed with individuals or in groups of two to three children. Interviewing children in pairs or small groups can help children to relax and keep each other on track and truthful (Graue \&Walsh, 1995).

The results from the behaviour mapping analysis and the analysis of the interviews suggested that the garden afforded the children more benefits than adversities and contributed to improved physical, cognitive and social functioning. The properties of the garden reduced the stress resulting in improved behavioural outcomes including:

i. Increases in vestibular movement and dexterity. (Movement generally involved with play activities). ii. Increases in ambulatory movement which improved children's physical fitness which was directly influenced by their illnesses or injuries.

iii. Increases in dexterity were seen in particular when children were engaged in social play.

\subsection{Identified Challenges}

In this section of the paper four key challenges will be discussed as they relate to both Said's (2008) and Bishop's (2008) studies. This will include the challenges of: ethics, access and consent; supporting the participation of children and young people in the research; being perceived as an outsider; and working within the culture of a hospital.

\subsection{Ethics, access and consent}

Processes such as obtaining ethics approval, recruitment and consent have enormous power over the research that is conducted and power over which groups of children can participate and in what capacity. Obtaining ethical approval for qualitative studies in health care contexts can be a battle with ethics bodies. Reaching a compromise can require altering the fundamental characteristics of the project, such as the age of participants approached in the study, or the type of consent that is adopted and who has to give it. These changes can result in the exclusion of groups of children from participating in the research. In the case of Bishop's (2008) study, access proved to be the most contentious issue. Being an outsider and not employed in the hospital community led to a very arduous recruitment and consent process being designed before the ethics approval was given. Overall the study required seven levels of permission spanning the university, the hospital, several layers of internal permission and finally the families and children themselves. For every single interview completed, between 10-15 adults were approached to provide access to the child involved.

Said (2008) also faced challenges negotiating initial support for his project but having gained it, experienced continuous and cooperative support from the hospital management and nursing staff for the duration of the study. The director of the hospital was thankful to the researcher for building a garden for her hospital. She supported the researcher's 
application for approval from the Health Ministry of Malaysia to construct the garden and to conduct the experimental research. With the presence of the garden, the nurses commented that the children were more cooperative with such things as taking medication, as the children felt going to the garden was a terrific reward. The researcher also developed a rapport with the ward staff by joining them in the garden up-keep programme which was carried out on the last Saturday of every month. This was done five months before the data collection began to ensure the plants, particularly shrubs and groundcovers were matured enough for the experiment. The researcher also continued to participate in the programme after the research was completed. All of these steps ensured a strong support of the project from the hospital staff.

\subsection{Supporting the participation of children and young people in the research}

Children's engagement in research requires a substantial time investment to ensure they comprehend the research process and are truly 'on the journey' with researchers. In a health care context, the time constraints on opportunities to work with children and young people can compromise this time completely. In Bishop's (2008) study, the original methodology had anticipated continued involvement from participants beyond their initial interview which included meeting to talk through a summary of the interview as a memberchecking task and involvement of children and young people in the analysis process and the development of findings. In reality children's and young people's lives in hospital were so unpredictable that meeting with the researcher more than once was very difficult to organise and could not be managed consistently. This in effect undermined the integrity of the participatory methodology planned, to the point where it was altered which also altered the children's experience of participation, reducing it to a point of a single contact with each participant. Children and young people still remained involved in all the parts of the process as initially planned but it was not possible to work with the same group throughout the study.

In Said's (2008) study, where the research was centred in a particular place in the hospital environment, the children were motivated to participate in the research by their enthusiasm for the garden. Even though the length of stays was short (an average of 3.6 days) the affordances of the garden as a play space were strong enough for the children to become interested in participating in the research. The research revealed that the children viewed playing in the garden as an opportunity to be away from the monotony and restriction of the ward. Hence, being in the garden enabled them to avoid boredom, be cheerful, play with other patients or siblings and forget their worries. This natural inclination to enjoy being in the garden led to daily visits and helped to facilitate the process of the research and children's willingness to be part of the research. Children had repeated, relaxed contact with the researcher and the garden during their hospital admission, with each child being interviewed up to four times during their hospital stay. 


\subsection{Being perceived as an outsider}

Being perceived as an outsider has both its advantages and disadvantages. For Bishop (2008), the main disadvantage laid in the arduous recruitment and consent processes as have been discussed. The main advantage included perceived neutrality by participants who felt free to speak openly about their response to the hospital community and environment as the researcher was not conceived as being part of it. Bishop suspects that had she been a member of the medical community, children and young people would have been more reserved in their discussion of the experience of the hospital and its community, wary of possible repercussions.

For Said (2008), children's perception of the researcher as an outsider was distinctive during the first day of the investigation. However, seeing the researcher behaving as a gardener helped children to see him as part of the fabric of the hospital environment. Enabling children to use the tape recorder and digital camera and other tools of the research also enabled the researcher to build rapport and break down the strangeness of the research process. These steps created opportunities for children to get acquainted with the researcher within the second day of their meeting. Being perceived as a seamless part of the hospital environment was part of the success of a phenomenological approach, in order to develop a firsthand perspective on children's experience.

\subsection{Working within the culture of a hospital}

The healing and treatment of patients are necessarily the dominant business of a hospital. At all times the needs of any particular social research project are necessarily the lowest in the order of priorities in this environment because these projects contribute nothing to the central business of the hospital in the immediate sense. Whilst this in itself is not difficult for a researcher to accept, the reality of the implications of it on the ground can prove very challenging. To complete her study, Bishop (2008) had to drive for an hour and a half out to the hospital to recruit and conduct interviews. On a number of occasions, children who had been booked to carry out interviews were not available as scheduled, due to unforeseen medical treatment, or because the patient had been granted leave unexpectedly, or had been discharged early. As the research had no particular priority or profile for anyone else in the hospital community, the researcher was never notified of such changes. This resulted in a number of unnecessary visits to the hospital. Whilst the hospital community was very welcoming to the researcher, at no point did the researcher feel a sense of belonging, or even of being a part of that community because of the dominance of the medical culture which transcended all relationships and identities within the environment, casting the social researcher as 'other' and 'outsider'.

When interviews were conducted, it was very difficult to find a quiet place within the ward environment or to give participants the opportunity to choose where they were interviewed. If the interviews were carried out at the patient's bedsides, then it became very difficult to protect the space for the duration of the interview, with one interview being interrupted 12 times in 30 minutes by medical staff with a need to check or see the patient. 
Similarly, Said (2008) had to drive one and half hours to the hospital to reach the hospital as early as the ward staff. The researcher had to water the plants early before the tropical sun rose, to minimise evaporation. On some occasions, children who were scheduled for interviews were discharged without prior notice to the researcher. In research on gardens as restorative environments, the major hurdle for the researcher was to obtain the research grant to finance the construction of the garden and the research. In Malaysia, research in health care settings is generally focused on pathogenic recovery methods rather than salutogenic (psychosocial) outcomes. This means that research funding for finding cures is considered more important than research that aims to understand and support the psychosocial needs of the patients. Thus, the continuity of research on children's restorative environments involving hospital gardens and landscape is likely to face difficulty.

\subsection{A Summary Discussion}

In Bishop's (2008) study the major considerations that truly challenged the researcher and the research were organisational and methodological. This began with the lack of familiarity of the hospital's ethics committee with qualitative research; the layers of gatekeepers and permission required to access children and young people; the arduous recruitment and consent processes; the lack of opportunities to follow up with children; and the inconsequence of the research to the immediate and dominant medical agenda. In total all these challenges threatened the capacity to complete the social research in medical contexts and also threatened to undermine the experience of participatory research for the children who become involved. Which groups of children and in what ways they can participate, are all determined by this context and this greatly inhibits individuals' capacity to choose to be part of such research projects as well as conditioning the type of experience that it will be for them in the end. Even though overall, Bishop would describe the hospital community as being very supportive of her and of the research during its course, she found it very difficult to practice and protect the tenets of participatory research and its values in this context.

In Said's (2008) study the major challenge was to build rapport with the hospital's administrators and caregivers in order to build cooperation and support for the research on children's recovery using a garden as a medium to foster recovery. It took many meetings with caregivers, doctors and administrators of the hospital to recognise the role of the garden as a restorative area for recovery. Said experienced very little acknowledgment or recognition from doctors who did not believe that the garden could help to enhance or foster the process of restoring the children's health. This reticence reflects an acknowledged resistance experienced by social researchers working in the context of health care settings for research questions and research that falls outside the central scientific questions associated with improving medical treatment.

Completing participatory research in a health care context is laced with challenges at 
many levels. These challenges act as a barrier to completing this kind of research in this context for researchers, yet the potential potency of the experience of hospitalisation in children's lives makes it imperative that we continue to hear their experience and remain grounded in our understanding of it.

\subsection{Recommendations And Conclusions}

The value of participatory research to our knowledge of children and childhood is supported by research in many disciplines. It is no less valuable in understanding the experience of health care contexts from children's and young people's perspectives. The reality of completing social research in a medical context is very difficult but should not be used to justify or prevent this kind of research from being completed. Without it, health care policies, health service provision and health care environments will be developed based on adults' assumptions about who children are and what their needs are. It is unlikely that the resulting products, services and environments will be as supportive and effective in meeting the needs of young people without knowledge of their experience. Bringing the views of children and young people to the decision-making processes that affect their lives reflects their rights as social citizens and grounds adults' understanding of children's experience in the reality of that experience.

In relation to improving the experience of completing a social research in a health care context, it is difficult to make any recommendation that is useful across all participatory study methodologies and methods. It is easy to identify the challenges but difficult to solve them as many of the difficulties are linked to working within the culture and context of another professional culture, which will inevitably impact the experience and over which the researcher has no control.

Some things are likely to change with time. Ethics committees are likely to get more familiar and comfortable with qualitative research as they are increasingly exposed to it; and medical audiences are also likely to become more receptive to input from qualitative research as they experience the benefit of that knowledge though their professional practice. Researchers also, are likely to develop a greater understanding of how to work within this culture and context, improving their capacity to preserve the tenets of participatory research and the experience for the children and young people involved.

\section{Acknowledgements}

Bishop would like to thank the children and young people who participated in this study for sharing their experience with the researcher; and the hospital community at the Children's Hospital at Westmead for their assistance in completing this study.

Said would like to thank the director and paediatric ward staff of Batu Pahat Hospital for their support in collecting the data for the research as well as in maintaining the garden as it 
is suitable for the children to play in it.

\section{References}

Alderson, P. (1993). Children's consent to surgery. Buckingham, UK: Open University Press. Alderson, P. (1995). Listen to children. London: Barnardo's. Bishop, K.G. (2008). From their perspectives: Children and young people's experience of a paediatric hospital environment and its relationship to their feeling of well-being.

The University of Sydney, Sydney, Australia. Bricher, G. (1999). Paediatric nurses, children and the development of trust. Journal of Clinical Nursing, 8: 451-458. Bricher, G. (2000). Children in hospital: Issues of power and vulnerability. Paediatric Nursing, 26(3): 277-282.

Carney, T., Murphy, S., McClure, J., Bishop, E., Kerr, C. \& Parker, J. (2003). Children's views of hospitalization: An exploratory study of data collection. Journal of Child Health Care, 7(1): 27-40.

Creswell, J. W. (1998). Qualitative Inquiry and Research Design: Choosing Among Five Traditions. Thousand Oaks, CA: Sage. Eiser, C. (2000). The psychological impact of chronic illness on children's development. In A. Closs (Ed.), The education of children with medical conditions. London: David Fulton.

Graue, M. E. \& Walsh, D. J. (1995). Children in context: Interpreting the here and now of children's lives. In: Hatch, J. A. ed. Qualitative Research in Early Childhood Settings, Westport: Praeger Publishers, 135-154.

Hutton, A. (2002). The private adolescent: Privacy needs of adolescents in hospitals. Journal of Pediatric Nursing, 17(1): 67-72.

Hutton, A. (2005). Consumer perspectives in adolescent ward design. Issues in Clinical Nursing, 14: 537-545.

Kesby, K. (2000). Participatory diagramming: Deploying qualitative methods through action research epistemology. Area, 32(4): 423-435.

Mauthner, M. (1997). Methodological aspects of collecting data from children: Lessons from three research projects. Children \& Society, 11: 16-28.

Miller, N. O., Friedman, S. B. \& Coupey, S. M. (1998). Adolescent preferences for rooming during hospitalization. Society for Adolescent Medicine, 23: 89-93.

Morison, M., Moir, J. \& Kwansa, T. (2000). Interviewing children for the purposes of research in primary care. Primary Health Care Research and Development, 1: 113

Said, I. (2008). Garden as restorative environment for hospitalised children. Malaysia: Penerbit, UTM Press.

Sandbaek, M. (1999). Adult images of childhood and research on client children. International Journal of Social Research Methodology, 2(3): 191-202.

Sartain, S. A., Clarke, C. L. \& Heyman, R. (2000). Hearing the voices of children with chronic illness. Journal of Advanced Nursing, 32(4): 913-921.

Smith, L. \& Callery, P. (2005). Children's accounts of their preoperative information needs. Journal of Clinical Nursing, 14: 230-238. 
Bishop, K., \& Said, I. / Asian Journal of Environmen-Behaviour Studies, ajE-Bs, 2(4) Jul / Sep 2017 (p.1-11)

Stalker, K., Carpenter, J., Connors, C. \& Phillips, R. (2004). Ethical issues in social research: Difficulties encountered gaining access to children in hospital for research. Child: Care, Health and Development, 30(4): 377383. 